\title{
Mountain Bike Rear Suspension Design: Utilizing a Magnetorheological Damper for Active Vibration Isolation and Performance
}

\author{
Robert Pierce, Sudhir Kaul, Jacob Friesen and Thomas Morgan \\ School of Engineering and Technology, Western Carolina University, Cullowhee, NC, 28723, USA.
}

\begin{abstract}
(Received 10 September 2019; accepted 29 August 2020)
This paper presents experimental results from the development of a rear suspension system that has been designed for a mountain bike. A magnetorheological (MR) damper is used to balance the need of ride comfort with performance characteristics such as handling and pedaling efficiency by using active control. A preliminary seven degree-of-freedom mathematical model has also been developed for the suspension system. Two control algorithms have been tested in this study - on/off control and proportional control. The rear suspension system has been integrated into an existing bike frame and tested on a shaker table as well as a mountain trail. Shaker table testing demonstrates the effectiveness of the damper. Trail testing indicates that the MR damper-based shock absorber can be used to implement different control algorithms. Test results indicate that the control algorithm can be further investigated to accommodate rider preferences and desired performance characteristics.
\end{abstract}

\section{INTRODUCTION}

The sport of mountain biking started in the 1970's when cyclists began to race recreational bicycles with oversized tires on mountain trails. ${ }^{1}$ In the 1980 's, bicycle manufacturers began to design and manufacture bikes specifically for mountain biking, and in the 1990's the first modern bicycle suspensions were incorporated into mountain bike designs. Since that time, mountain biking has grown significantly in popularity and has become one of the two major genres of cycling. Commercial bicycles designed specifically for mountain biking these days come in many different types, each design typically caters to a specific riding style such as downhill riding, cross country racing, etc.

Mountain bikes are most commonly designed with either a front suspension only or a dual system consisting of the front and rear shock absorbers, the latter is commonly referred to as a "full suspension" bike. The front suspension of either design almost always consists of a pair of parallel, translational joints incorporated into the two fork tubes. These joints utilize some combination of mechanical springs, gas springs, and hydraulic dampers. The front suspension design most often contains an independently-adjustable compression and rebound response.

Unlike front suspensions, rear suspension designs vary significantly from one manufacturer to another. Rear suspensions typically utilize a rear triangle that holds the rear wheel axle that can pivot relative to the main frame tubes. Motion of the rear triangle is controlled through a kinematic linkage and a shock absorber. The linkage geometry and the location of the shock absorber vary with the intended application and the design adopted by the bike manufacturer. Like the front system, rear shock absorbers typically consist of some combination of mechanical springs, air springs, and hydraulic dampers, often with independent compression and rebound control. Using a rear suspension system offers two primary advantages: 1) the rider experiences lower impact forces when travelling over rough terrain, thereby reducing rider fatigue, and 2) the rear wheel of the bike maintains contact with the trail as it trav- els over a bump, resulting in improved handling. Maintaining contact between the rear wheel and the trail is particularly important when the rider is climbing through rough terrain to maintain traction and, thereby, control the bike.

While full suspension bikes offer advantages, it has been suggested that they are less efficient than bikes with only a front suspension due to increased energy lost to damping in the rear shock. ${ }^{2}$ Several studies in the literature have examined this research question, with varying results. ${ }^{3-5}$ While full suspension bikes may be less efficient at transforming energy at the pedals into potential and kinetic energy of the bike, a substantial enhancement of rider comfort and control appears to offset these losses in mechanical efficiency so that the overall efficiency of the rider/bicycle system is not appreciably different between a full suspension design and a design with front suspension only.

Full suspension designs are required to balance the need for pedaling efficiency, which calls for a stiff suspension, with comfort, which calls for a soft suspension. Furthermore, bike racers and riders of higher-end bikes desire a suspension with a highly customizable response that can be tuned for different riding styles and conditions. In order to meet these requirements, manufacturers have started to design bikes with semi-active suspension systems. The first such design became commercially available in 2002 with a suspension that uses a hydraulic inertia valve that is mounted on the rear triangle, near the rear axle. ${ }^{6}$ Vertical acceleration of the rear wheel causes the valve to open so that fluid can flow between the reservoir in the valve and a hydraulic damper in the rear shock absorber, reducing the damping coefficient. The bike is set up so that the frame is normally quite stiff, with relatively high pedaling efficiency. When the rear wheel hits a bump or rough terrain, the rear suspension becomes softer as the inertia valve senses higher acceleration of the rear axle. After the bike passes through the bump or rough terrain, the suspension returns to the default stiffer setting.

A more recent design, which became commercially avail- 
able in 2018, utilizes electronic sensors, actuators, and a controller powered by a battery. ${ }^{7}$ Acceleration levels of the bike at the rear axle, seat tube, and front fork crown are measured using micro-electromechanical (MEMS) accelerometers mounted at these points. Accelerometer signals are sent to an onboard microcontroller which can change the damping characteristics of the rear shock absorber by using a servocontrolled valve. This system can detect different combinations of acceleration conditions (e.g. accelerations that correspond to being airborne after a jump) and set the rear shock absorber to a soft or stiff setting.

One approach to semi-active damping of mountain bike suspensions that was developed in the 2000's but has not been commercialized is the use of a magnetorheological (MR) damper as the shock absorber damping element. ${ }^{8-11}$ An MR damper is a hydraulic damper that is filled with magnetorheological fluid, the fluid consists of micron-sized ferromagnetic particles contained in a carrier fluid. ${ }^{12}$ When the fluid is exposed to a magnetic field, the ferromagnetic particles align themselves with the lines of flux of the magnetic field, in turn resulting in an increase in the apparent viscosity of the fluid. This results in an increase in the damping coefficient of the damper. The main advantage of MR dampers is that the damping coefficient can be actively and reversibly controlled by controlling the intensity of the magnetic field applied to the fluid. Due to its inherent nonlinear and hysteretic characteristics, several models have been proposed to represent the MR fluid's stress-strain behavior as well as the damping characteristics as the intensity of the magnetic field is varied. ${ }^{13,14}$ Furthermore, the use of MR damping has also been investigated in automotive applications. ${ }^{15}$

One characteristic of all the semi-active mountain bike suspension designs discussed above is that they utilize a fullyon/fully-off algorithm to control the state of the damper. All these designs use an acceleration threshold value and switch the damper between a soft setting and a stiff setting when the threshold is reached. It has been demonstrated that switching the MR damper current between no current and the saturation current does affect the frequency response, as measured at the seat post when the mountain bike is ridden on a trail. ${ }^{8}$ However, the previous work does not consider the use of alternative control strategies to tailor the response of the bicycle.

The work presented in this paper addresses two research questions: 1) Can a mountain bike rear shock absorber be designed to utilize an MR damper with a control algorithm that is more complex than a simple on-off controller? 2) How does a mountain bike that uses an MR damper-based rear shock absorber perform while responding to alternate control strategies? In Section 2, the development and characterization of a programmable, MR damper-based rear shock absorber is discussed. In Section 3, a preliminary planar model of a mountain bike is presented, this model is used to predict frequency response. Frequency response of an actual mountain bike using three different damper currents is measured in the laboratory as well as a mountain trail, these results are presented and discussed in Section 4. An on-off control algorithm and a proportional control algorithm have been implemented and the response using these two control strategies is measured on the mountain trail. The main conclusions of this study are presented in Section 5 along with a discussion of future research.

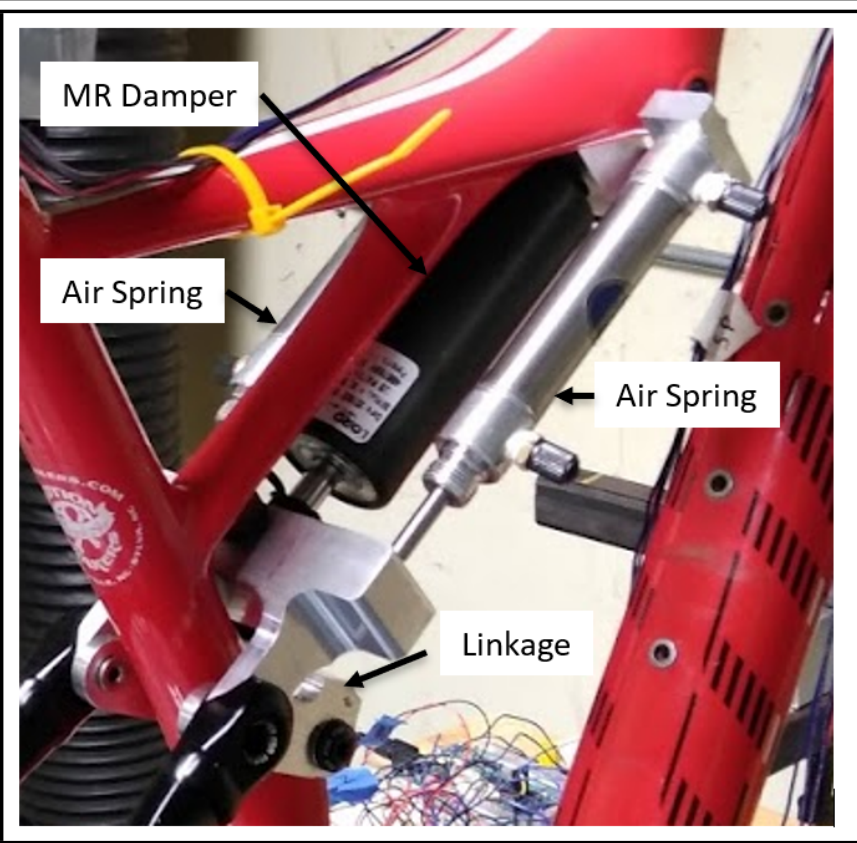

Figure 1. The MR damper-based rear mountain bike shock absorber.

\section{DESIGN AND EXPERIMENTAL STEP}

This section presents the overall design of the rear shock absorber developed in this study by using an MR damper. The experimental set up and the control strategy are also presented in this section.

\subsection{MR Damper-Based Shock Absorber - Design}

The MR damper-based shock absorber was incorporated into the rear suspension of a 2014 Specialized Epic with an aluminum frame. This is a "cross-country" bike that is designed to strike a balance between high pedaling efficiency and shock absorption on rough trails. The bike was originally equipped with the hydraulic inertia valve system described in the previous section to provide semi-active damping. Figure 1 shows the MR damper-based shock absorber, which was designed to fit into the suspension linkage without altering the kinematics of the linkage. In order to simplify integration and facilitate alterations, the shock absorber was developed by using a commercially-available MR damper (Manufacturer: Lord Corporation, Model: RD 8040-1). This is a piston-damper, with the coil located coaxially with the piston orifices. At a velocity of $0.2 \mathrm{~m} / \mathrm{s}$, the damper generates a force of $120 \mathrm{~N}$ at $0 \mathrm{~A}$ coil current and $1450 \mathrm{~N}$ at the saturation current. ${ }^{16}$ The shock absorber also uses a pair of air springs (Manufacturer: Bimba, Model: SR-042-DPY) that are mounted symmetrically, on either side of the damper. Each of the air springs has a separate compression and a rebound chamber and the pressure in these chambers can be set independently. The overall length of the shock absorber was determined by the suspension linkage, however the only constraint on the width of the shock absorber assembly was to maintain adequate clearance for the rider's legs. Since the primary aim of the design of the shock absorber was experimental, the system was not designed to minimize volume or weight at this stage.

A schematic diagram of the shock absorber system is shown in Fig. 2, and a picture of the full experimental set up is shown 


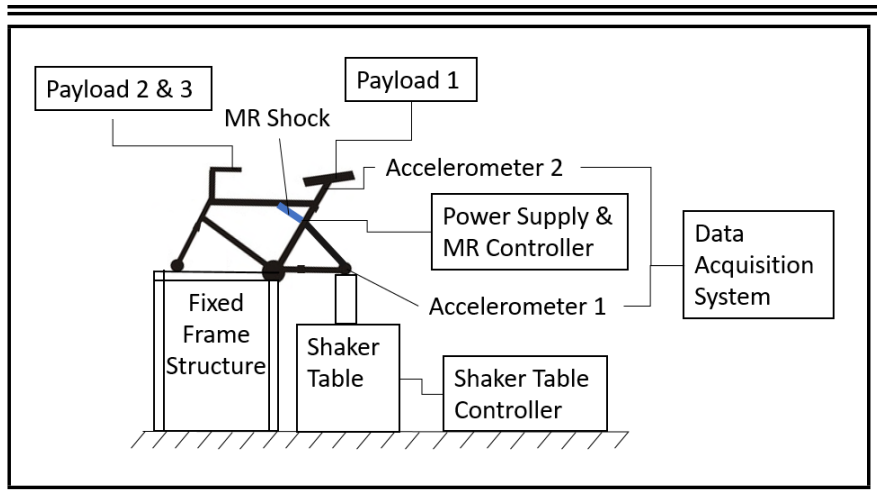

Figure 2. Schematic diagram of the shaker-table experiment.

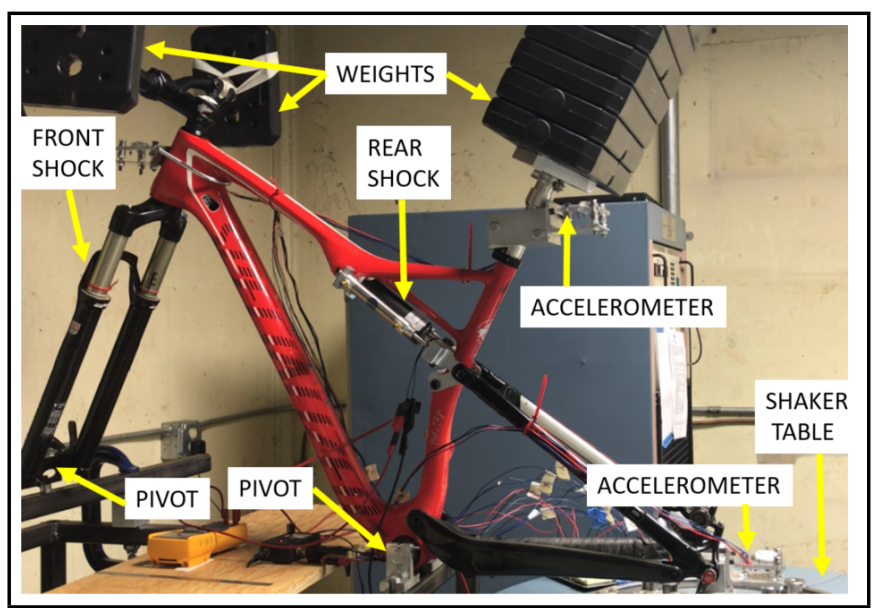

Figure 3. The shaker-table experimental set up.

in Fig. 3. The MR damper is a piston-based damper with a coaxial electromagnetic coil located near the piston orifices. Current through the coil can be varied continuously between 0 and 1.5 Amperes using a current amplifier (Manufacturer: Lord, Model: Wonder Box $\left.{ }^{\circledR}\right)$. This current creates a magnetic field around the piston orifices, increasing the apparent viscosity of the MR fluid and subsequently increasing the effective damping coefficient. Current to the damper coil is controlled by a 32 bit, Atmel SAM8XE microcontroller running at $84 \mathrm{MHz}$ and mounted on an Arduino Duo development board. It may be noted that the response time of the MR fluid can limit the controller's capability. 8,16 The MR coil current is set by the microcontroller by assigning a value of an unsigned integer, referred to as the "shock number". The value of the shock number is written to a Digital to Analog converter (DAC) which converts the number to an analog control voltage between 0 and 5 volts. This voltage is used as a control signal to the current amplifier which outputs a current that is proportional to the control voltage. Inputs to the microcontroller are provided by 3-axis MEMS accelerometers (Manufacturer: Analog Devices, Model: ADXL335) that are mounted near the rear axle and on the seat post. These accelerometers can be used strictly for data acquisition when the system is run with a constant damper current or they can be used for both data acquisition as well as control inputs when the system is running in a feedback control mode.

\subsection{Shaker Table Testing}

To investigate the bike response to the MR damper-based shock absorber, the original rear suspension was removed and replaced with the MR rear shock absorber system, as shown in Fig. 1. A new set of rear suspension links was designed to assemble the MR shock absorber to the bike. These links were designed so that the linkage had the same kinematics as the original linkage. The bike was mounted to a fixture that was fabricated from steel tubing. The bottom bracket of the bike was mounted to the fixture so that the frame could not translate but would still have the ability to rotate (pitch of sprung mass) about the axis of the bottom bracket, as can be seen from Fig. 3. The front fork of the bike was mounted to the fixture using the front axle mount so that the front fork could move through its travel but the axis of the front axle could not translate. The rear axle mount was attached to the actuator platform of a single degree-of-freedom shaker table (Manufacturer: Unholtz Dickie, Model: S452 LP). The frame was oriented such that the vertical motion of the shaker remains perpendicular to the bottom links of the rear triangle.

The interaction of forces between a mountain bike rider and the bike is complex and difficult to simulate in a laboratory setting. In the shaker table test set up, external forces have been applied to the bike at the rear axle by the shaker, at the bottom bracket by the fixture mount, at the front axle by the fixture mount, and by weights mounted at the seat post and on the handlebars. The seat post weights had a mass of $39.7 \mathrm{~kg}$, and each handlebar weight had a mass of $3.2 \mathrm{~kg}$. The distribution of mass at different locations of the bike was found from the literature for a typical bicycle rider. ${ }^{17}$

The air spring in the front fork of the bike was pressurized to $480 \mathrm{KPa}$, which is listed by the manufacturer as the appropriate pressure for a rider with the mass distribution described above. Pressure in the air springs of the MR shock absorber assembly was set by watching the compression of the suspension as the weights were applied and using a pressure that put the shock at the middle of its stroke. This approach is commonly used to set up a mountain bike suspension for a particular rider. This resulted in a pressure of $550 \mathrm{KPa}$ in both the compression and rebound sides of the cylinders. It may be noted that while the distribution of rider mass and inertia of a rider can have a significant influence on the dynamic response, ${ }^{17}$ the use of a particular rider mass distribution is not expected to restrict the application of this work in any manner. The use of adjustable air springs and dampers in existing mountain bike suspensions makes the system "tunable" to a variety of riders, the larger goal of this research is to enhance suspension tuning.

\subsection{Suspension Control and Data Acquisition}

Data acquisition and control of the damper current was accomplished through the Atmel SAM8X microcontroller. Acceleration was measured at the rear axle and just behind the seat post of the bike by using three-axis MEMS accelerometers. Acceleration values were measured every 5 milliseconds using the 10-bit A to D converters on the microcontroller. Acceleration data were recorded onto a microSD card using a serial-peripheral-interface (SPI) bus. Current through the MR damper was controlled by using the microcontroller, a D to A converter (DAC), and the MR damper current amplifier. The microcontroller code generates an integer that is proportional to the desired coil current. The DAC converts this number to a DC voltage that is used as the control signal to the current 
amplifier.

The response of the MR shock absorber was measured on the shaker table by using a series of constant, sinusoidal inputs and by using a sine sweep input. The set frequencies were $2,5,10$, and $20 \mathrm{~Hz}$ while the sine sweep of 1 to $20 \mathrm{~Hz}$ over 20 seconds was used. Each input condition was run at damper currents of $0.0 \mathrm{~A}, 0.4 \mathrm{~A}$, and $1.4 \mathrm{~A}$. The 0.0 A current results in the softest setting of the damper while the $1.4 \mathrm{~A}$ current results in magnetic saturation, thereby providing the stiffest setting. The mid-range current of $0.4 \mathrm{~A}$ was selected by experimenting with the system to find a current at which the displacement of the shock absorber under different loading profiles was found to be near the midpoint between the displacement at the $0.0 \mathrm{~A}$ and 1.4 A settings.

\subsection{Trail Testing}

Since the interactions between the trail, the bike, and the rider are complex and difficult to reproduce in the laboratory, some testing has been conducted on a mountain trail to test the control algorithm while measuring accelerations. The bike was removed from the shaker table and re-assembled with wheels and necessary drive components. Wires were routed from the accelerometers and from the MR damper to a backpack that held the microcontroller board and peripherals, the current amplifier, and a 2 Ah, 24 V DC battery (Manufacturer: Kobalt, Model: KB 224-03) that was used to power the controls and to supply current to the MR damper. A backpack was worn by the rider to carry all the above during testing, as shown in Fig. 4. The rider is an experienced mountain biker with a mass of $86 \mathrm{~kg}$. Pressure in the front shock air spring was set to the manufacturer's recommendation of $820 \mathrm{KPa}$ during the trail testing. Pressure in the rear air springs, which was set by measuring the compression of the rear shock under the rider's weight, and set to $890 \mathrm{KPa}$ in both the compression and rebound chambers.

Acceleration data was collected on a rocky trail with a moderate downhill slope and a surface of hard-packed dirt with gravel and rocks. Data was collected with the damper current at $0.0 \mathrm{~A}, 0.4 \mathrm{~A}$, and $1.4 \mathrm{~A}$. Trail tests were also performed using an on-off control algorithm and a proportional control algorithm. A general block diagram of the control system is shown in Fig. 5. The control algorithm for the on-off control is governed by the following logic:

if $\left(\left(a_{n}+a_{n-1}\right)>t_{u o}\right.$ OR $\left.\left(a_{n}+a_{n-1}\right)<t_{l o}\right)$ then $s=s_{s o f t}$ else $s=s_{\text {stiff }}$.

In Eq. (1), $a_{n}$ is the $n^{\text {th }}$ data point of the rear-axle accelerometer z-component and $a_{n-1}$ is the $(n-1)^{t h}$ data point of the rear-axle accelerometer z-component, $t_{u o}$ is the upper threshold for the sum of two acceleration readings for the on/off controller, $t_{l o}$ is the lower threshold for the sum of two acceleration readings for the on/off controller, $s$ is the shock number that sets the current in the MR damper, $s_{\text {soft }}$ is the shock number corresponding to no current $(0.0 \mathrm{~A})$, and $s_{\text {stiff }}$ is the shock number corresponding to saturation current (1.4 A).

The control algorithm uses the sum of two consecutive acceleration measurements to mitigate the probability that the

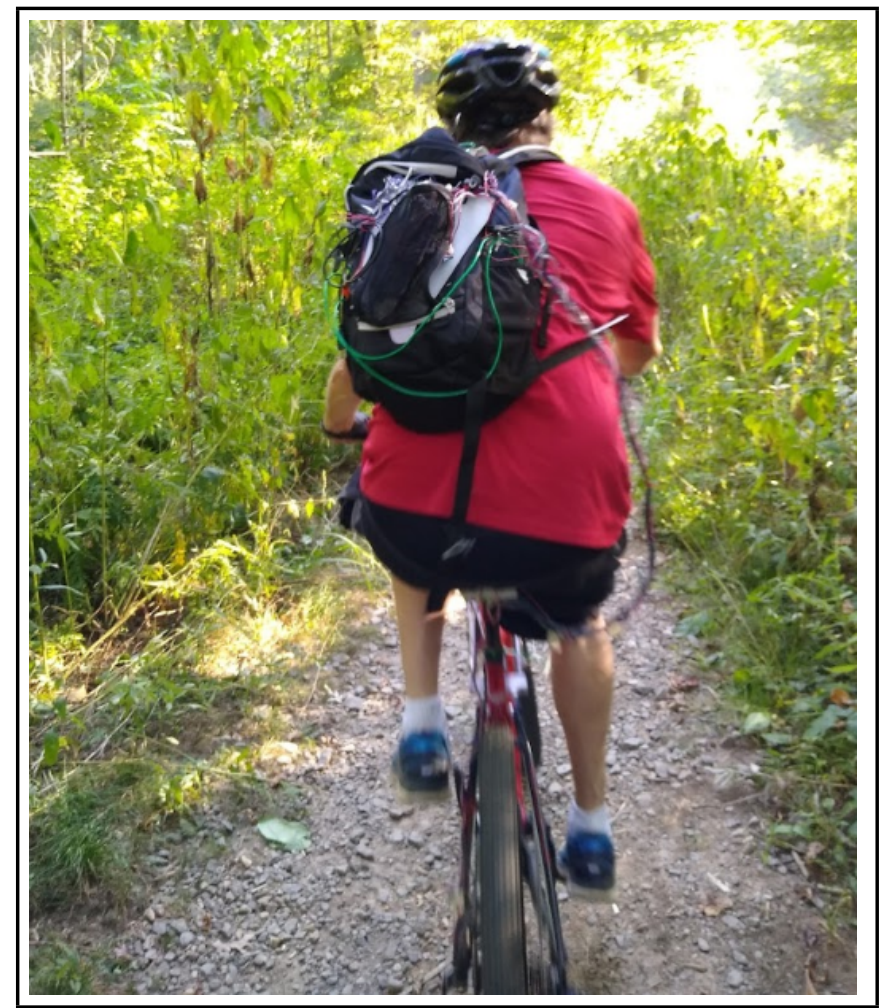

Figure 4. The MR shock-equipped bike on the trail.

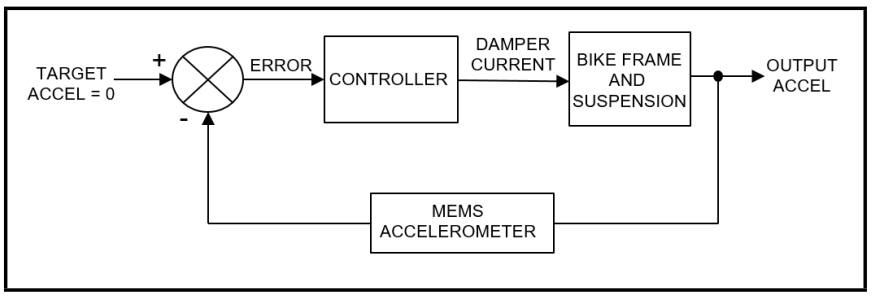

Figure 5. Block diagram of the closed-loop damper control system.

controller will react to noise in the measurement. The absolute value of the sum is used since accelerations can be up or down. When the sum of the two accelerations is below the threshold value the shock is in its stiffest possible state to improve handling and pedaling efficiency. When the sum of accelerations exceeds the threshold, the shock is switched to its softest possible state, which should result in higher ride comfort. The threshold values for the on-off controller were set to correspond to an acceleration of $\pm 8.1 \mathrm{~m} / \mathrm{s}^{2}$ (approximately $0.8 \mathrm{~g})$.

The control algorithm for proportional control is governed by the following logic:

$$
\begin{aligned}
& \text { if } \left.\left(e>t_{u p} \text { OR } e<t_{l p}\right) \text { then } s=s_{\text {stiff }}-m * e\right) \\
& \text { else } s=s_{\text {stiff }} .
\end{aligned}
$$

In Eq. (2), $e=a_{0}-\left(a_{n}+a_{n-1}\right), a_{0}$ is the absolute value of two consecutive accelerometer readings when rear axle acceleration is zero, $t_{u p}$ is the upper threshold for the sum of acceleration readings, and $t_{l p}$ is the lower threshold for sum of two acceleration readings for the proportional controller. The goal of the controller is to minimize acceleration. A threshold value of $3.8 \mathrm{~m} / \mathrm{s}^{2}$ is used to reduce the effects of noise. If acceleration is below the threshold, the shock is set to its stiffest state. When the acceleration exceeds the noise threshold, the 


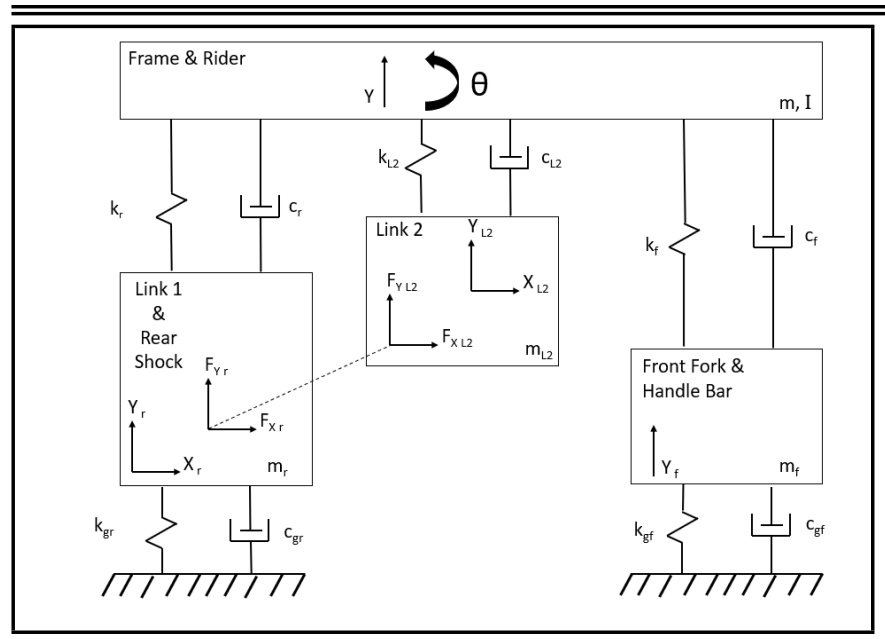

Figure 6. Seven degree-of-freedom model.

current through the MR damper is reduced in proportion to the acceleration to enhance ride comfort.

All acceleration values are initially stored as an array of integers from the microcontroller A to D converters. The arrays are then post-processed in MATLAB $®$ in order to convert them into acceleration values with the appropriate units of $\mathrm{m} / \mathrm{s}^{2}$.

\section{MODEL}

A preliminary mathematical model has been developed to represent the in-plane dynamics of the mountain bike. Some of the test results are compared with the results of the model to gain an insight into the suspension of the mountain bike. This section presents the mathematical model and the main results obtained from this model.

The model has been divided into four rigid bodies with seven degrees-of-freedom overall, as shown in the form of a schematic in Fig. 6. The four rigid bodies in the model in Fig. 6 consist of the seat stay frame link (Link 1), the chain stay frame link (Link 2), the bike frame and rider, and the front fork with handle-bars. It may be noted that the model assumes that the rider is rigidly attached to the frame. This is an approximation since it does not represent any compliance as well as any change of position of the rider, but this represents the test set up used for the shaker table testing for this study. Therefore, the sprung mass $(m)$ consists of the frame and a portion of the rider mass. The remaining portion of the rider mass is assigned to the front fork $\left(m_{f}\right)$. The unsprung mass of the front and rear wheels is added to $m_{f}$ and $m_{r}$ respectively. These unsprung masses can be updated along with the stiffness of the front and rear tires. The sprung mass has been modeled with two degrees-of-freedom - bounce and pitch - while Link 1 and Link 2 have been modeled with fore-aft and vertical degrees-of-freedom. The front fork has been modeled with one degree-of-freedom only.

The governing equations of motion for this model have been adapted from another study in the literature to derive the transmissibility equation. ${ }^{18}$ For the model shown in Fig. 6, the displacement transmissibility due to external excitations can be expressed as:

$$
T=\left[-\omega^{2} M+i \omega C+K\right]^{-1}\left[K^{\prime}+i \omega \dot{C}\right] .
$$

In Eq (3), $T$ is the $7 \times 1$ transmissibility matrix while $\omega$ is the excitation frequency, and $M, C, K$ are the mass, damping

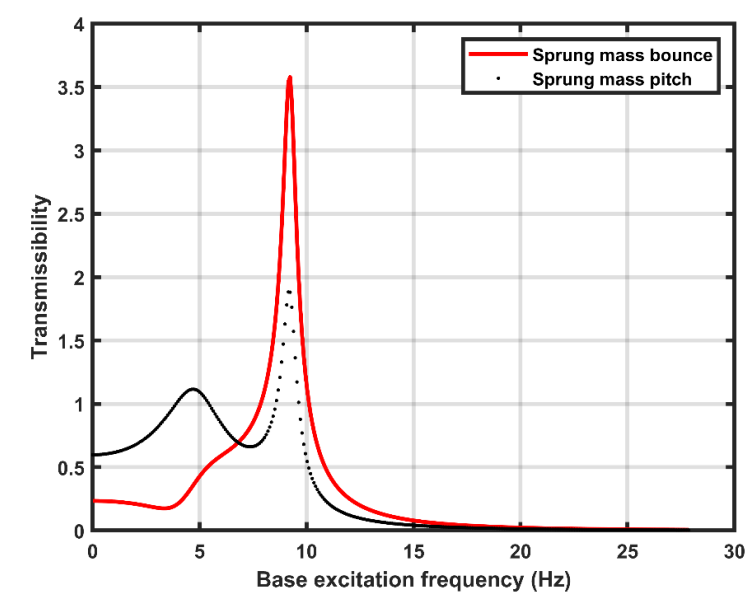

Figure 7. Displacement Transmissibility - Sprung Mass Bounce and Pitch Excitation Input at Rear Wheel.

and stiffness matrices respectively. Furthermore, $K^{\prime}$ and $\dot{C}$ are $7 \times 1$ matrices resulting from the source of base excitation. The frequency response function (FRF) for accelerations pertaining to all degrees-of-freedom of the model can be expressed as follows:

$$
H(\omega)=-\omega^{2}\left[-\omega^{2}+i \omega C+K\right]^{-1}[\hat{K}+i \omega \hat{C}] .
$$

In Eq. (4), $H(\omega)$ is the FRF matrix for the system that calculates accelerations for all degrees-of-freedom due to excitations at the front tire and the rear tire while $\hat{K}$ and $\hat{C}$ are $7 \times 2$ matrices. The FRF matrix can be used to compute the modified FRF, $H^{*}(\omega, V)$, that accounts for wheelbase filtering corresponding to the constant speed, $\mathrm{V}$, at which the bicycle is traveling in a straight line. This is a constraint of the model since it can only represent planar motion of the bike.

The acceleration power spectral density (PSD) for each degree-of-freedom can be computed by using the modified FRF and the PSD of the road surface as:

$$
S_{i i}(\omega, V)=\left|H_{i}^{*}\right|^{2} S_{r r}(\omega, V) .
$$

In Eq. (5), $S_{r r}(\omega, V)$ is the PSD of the road profile while $S_{i i}(\omega, V)$ is the PSD of acceleration for each degree-offreedom of the model in Fig. 6. The use of PSD is common in ride comfort and handling analysis of automobiles with a welldocumented list of different road surfaces. ${ }^{19}$ However, most of the surfaces documented in the literature represent paved roads and some gravel roads whereas the off-road terrain is the primary surface of interest for mountain bikes. The off-road terrain can vary significantly from gravel roads to mountain trails. The International Organization for Standardization (ISO) classifies road surfaces based on the PSD of road profiles with an index-based road classification. ${ }^{19}$ Based on this road classification, only Class C (highway with gravel) and Class D (rough runway) profiles have been used to simulate the input to the rear axle of the mountain bike.

Figure 7 shows the displacement transmissibility of the sprung mass when the rear wheel is excited and when there is no current input to the rear shock. As can be seen from Fig. 7, the bounce and pitch modes are coupled with distinct peaks at around $4 \mathrm{~Hz}$ and $9 \mathrm{~Hz}$. 


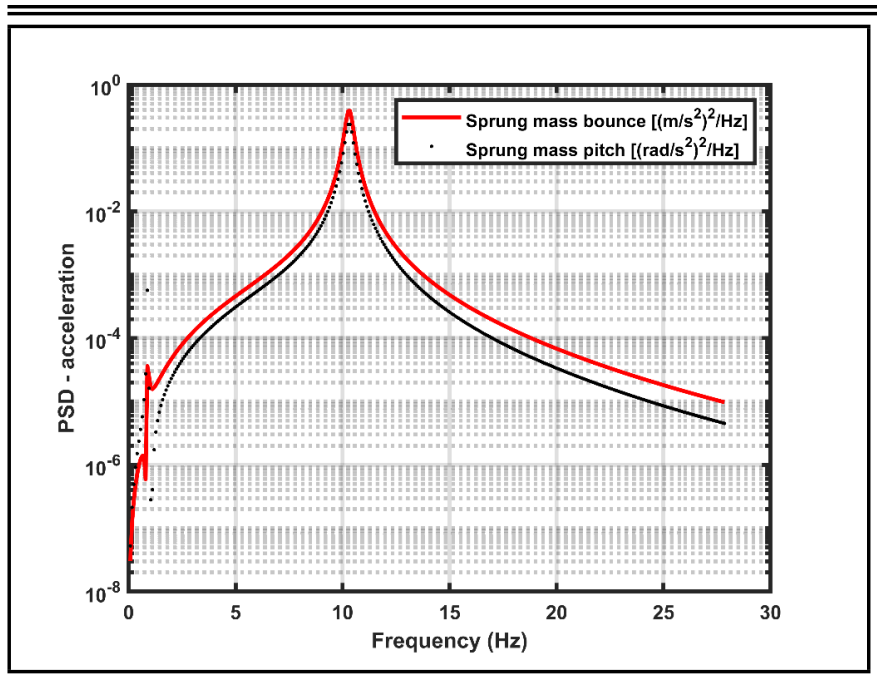

Figure 8. Power Spectral Density - Acceleration - Sprung Mass.

Figure 8 shows the PSD plot for acceleration of the sprung mass when the bike is traveling at a constant velocity of $4 \mathrm{~m} / \mathrm{s}$ on a relatively rough road surface (Class D as per ISO) while the damper in the rear shock system is at the saturation current. Distinct peaks can be observed at $1 \mathrm{~Hz}$ and $10 \mathrm{~Hz}$ with very similar bandwidths for pitch and bounce at $10 \mathrm{~Hz}$. It can be observed that at around $1 \mathrm{~Hz}$, the pitch acceleration exhibits a relatively higher bandwidth.

As discussed earlier, this model assumes a rigid connection between the frame and the rider while the rider is in an upright position. However, the model presented in this section can accommodate different distribution of the rider mass and inertia due to multiple rider positions in order to comprehend variability and sensitivity. The literature on bicycle dynamics provides guidelines about possible means of distributing a rider's mass and inertia from the torso, arms and legs. ${ }^{17}$

The preliminary model presented in this section can be used as a baseline to comprehend the sensitivity of the response to different designs of the suspension system or to determine the mountain bike's performance on different trails while traveling in a straight line. It may, however, be noted that this model is a first attempt and needs to be developed further to account for suspension kinematics as well as an appropriate distribution of rider mass and inertia under different riding conditions. The results of the model will be compared with the experimental results in the subsequent section.

\section{RESULTS}

This section presents the results from shaker table testing as well as trail testing at different settings of the MR damper. Some results from the implementation of the control algorithms are also presented in this section. Furthermore, some of the test results are compared with the results of the theoretical model.

The RMS (root mean square) acceleration at the rear axle and the seat post from the shaker table testing is shown in Fig. 9. Acceleration values are shown for damper currents of $0.0 \mathrm{~A}, 0.4 \mathrm{~A}$, and $1.4 \mathrm{~A}$ with input excitation frequencies of $2,5,10$, and $20 \mathrm{~Hz}$. At the $2 \mathrm{~Hz}$ input frequency, the effect of stiffening the damper by increasing the damper current can be clearly observed in Fig. 9(a). As the current is increased from the softest $(0.0 \mathrm{~A})$ setting to the stiffest $(1.4 \mathrm{~A})$ setting, (a)

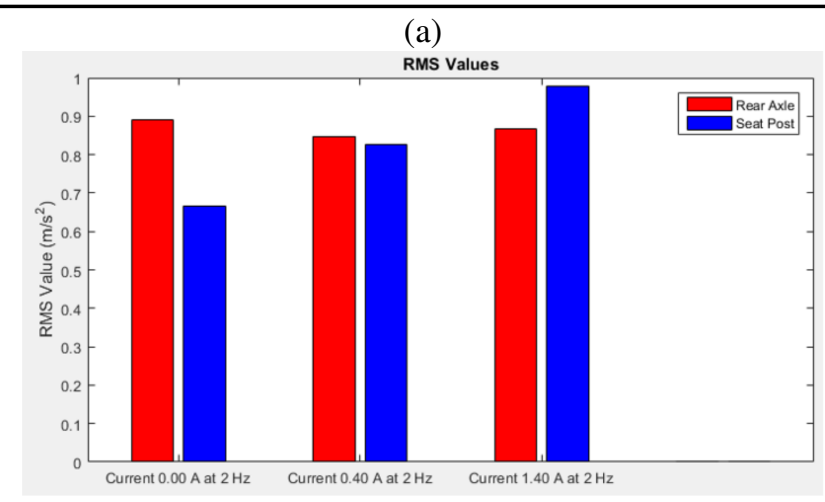

(b)

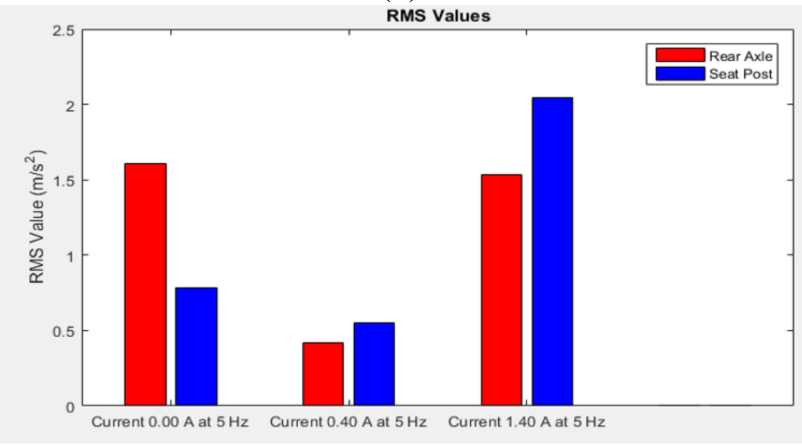

(c)

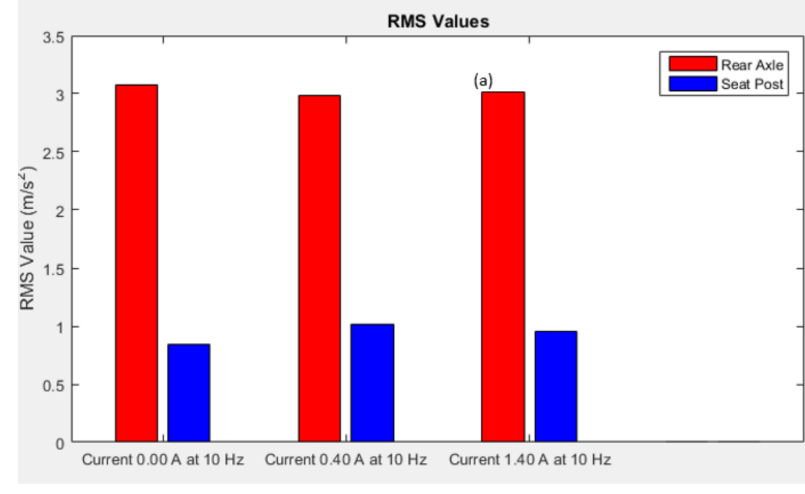

(d)

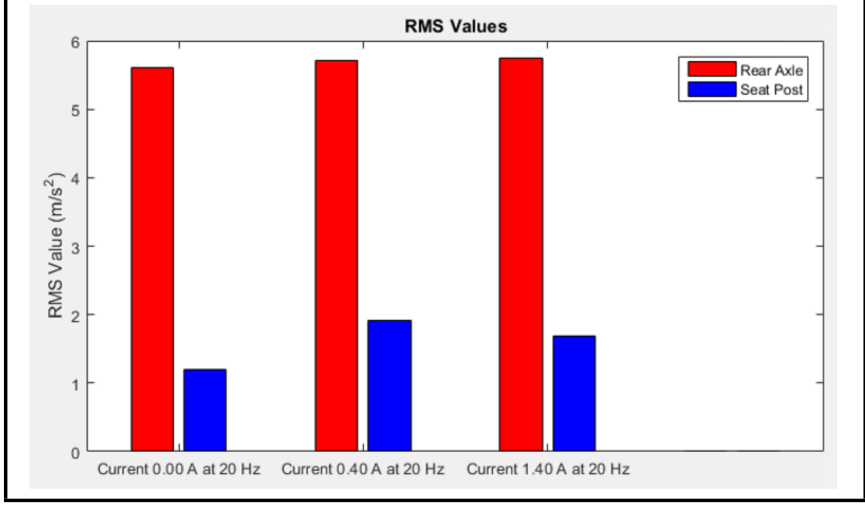

Figure 9. RMS accelerations measured on the shaker table using damper currents of 0.0, 0.4, and 1.4 A. Measurements at driving frequencies of (a) $2 \mathrm{~Hz}$, (b) $5 \mathrm{~Hz}$, (c) $10 \mathrm{~Hz}$, (d) $20 \mathrm{~Hz}$.

the acceleration at the seat post keeps increasing. It may be noted that the acceleration at the mid-range damping current of $0.4 \mathrm{~A}$ is between the accelerations at the softest and stiffest settings. This indicates that at a relatively low frequency, it may be possible to control the response of the shock absorber by using a proportional control algorithm.

At $5 \mathrm{~Hz}$, the response of the system to increasing damper 


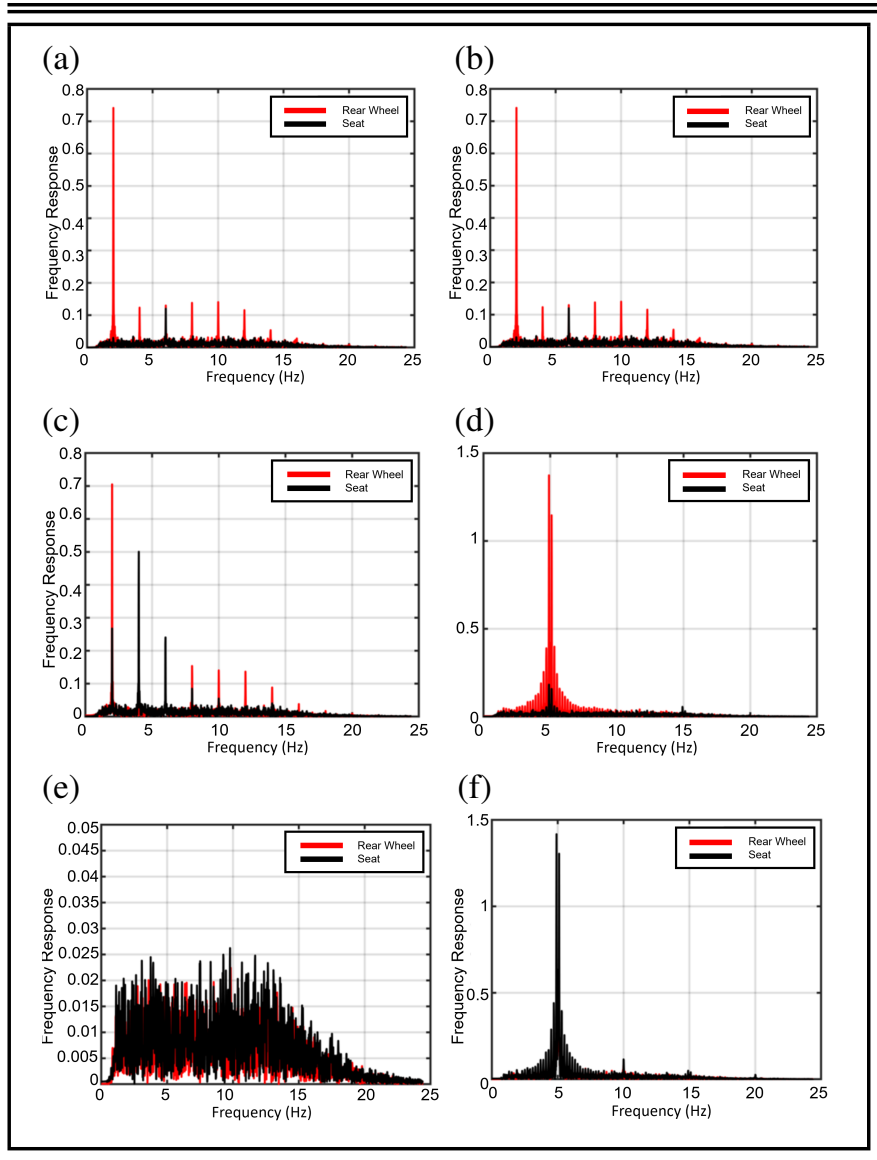

Figure 10. Frequency response of acceleration at excitation frequencies of $2 \mathrm{~Hz}$ and $5 \mathrm{~Hz}$. (a) Frequency $=2 \mathrm{~Hz}$, Current $=0.0 \mathrm{~A}$, (b) Frequency $=2 \mathrm{~Hz}$, Current $=0.4 \mathrm{~A}$, (c) Frequency $=2 \mathrm{~Hz}$, Current $=1.4 \mathrm{~A}$, (d) Frequency $=$ $5 \mathrm{~Hz}$, Current $=0.0 \mathrm{~A}$, (e) Frequency $=5 \mathrm{~Hz}$, Current $=0.4 \mathrm{~A}$, (f) Frequency $=5 \mathrm{~Hz}$, Current $=1.4 \mathrm{~A}$.

current is very different from the response at $2 \mathrm{~Hz}$. As the shock absorber becomes stiffer, the acceleration at the seat post drops initially and then increases significantly. One possible explanation for this behavior can be found from the response in Fig. 10, which shows the frequency response plots for the tests conducted at all three levels of damper current. At $2 \mathrm{~Hz}$, increasing the damper stiffness increases the relative magnitude of the response at the seat post but the frequencies that are excited do not change. When the input frequency is at $5 \mathrm{~Hz}$, the response at the seat post is damped when the damping current is $0.0 \mathrm{~A}$. As the current is increased and the damper becomes stiffer, there is a resonance at $5 \mathrm{~Hz}$ and the relative magnitude of the seat post response is higher as compared to the input at the rear axle. This resonance at $5 \mathrm{~Hz}$ can also be clearly discerned from Fig. 11, which shows the frequency domain results for a sine sweep input of $1-20 \mathrm{~Hz}$ at the rear axle. As the damper current is increased, the relative magnitude of the response at the seat post is seen to increase in Figure 11, particularly near the $5 \mathrm{~Hz}$ resonance. This could be attributed to the frame assembly and the structure holding the seat post on the bike.

At the higher input frequencies of $10 \mathrm{~Hz}$ and $20 \mathrm{~Hz}$, changes in the damper stiffness do not appear to have much effect on the response of the bike at the seat post. As can be seen from Fig. 11, the damper is still successful in providing isolation at the seat post, however the response does not change significantly with the increase in the damper current.

Figure 12 shows the RMS accelerations at the rear axle and

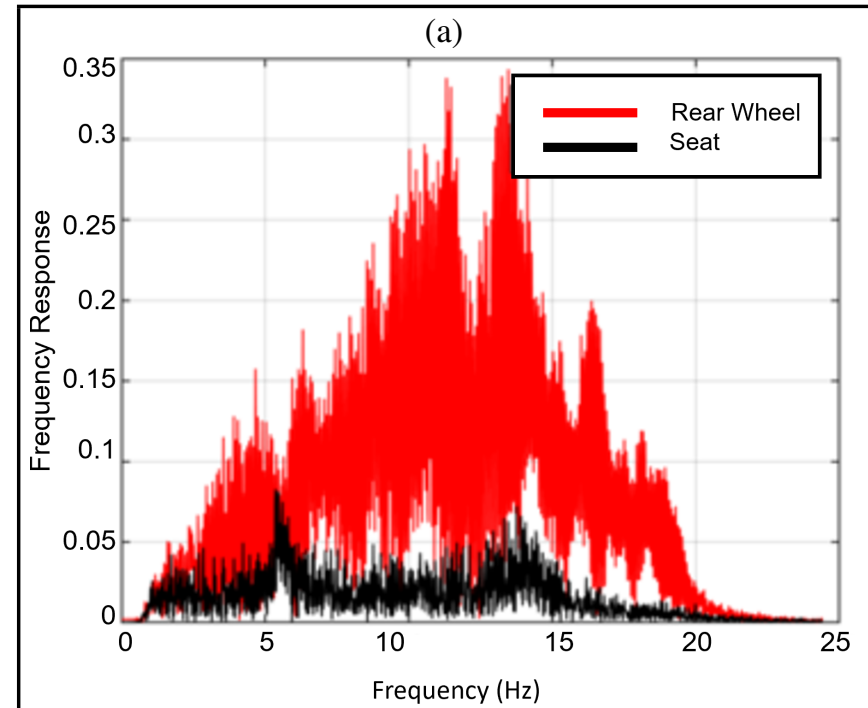

(b)

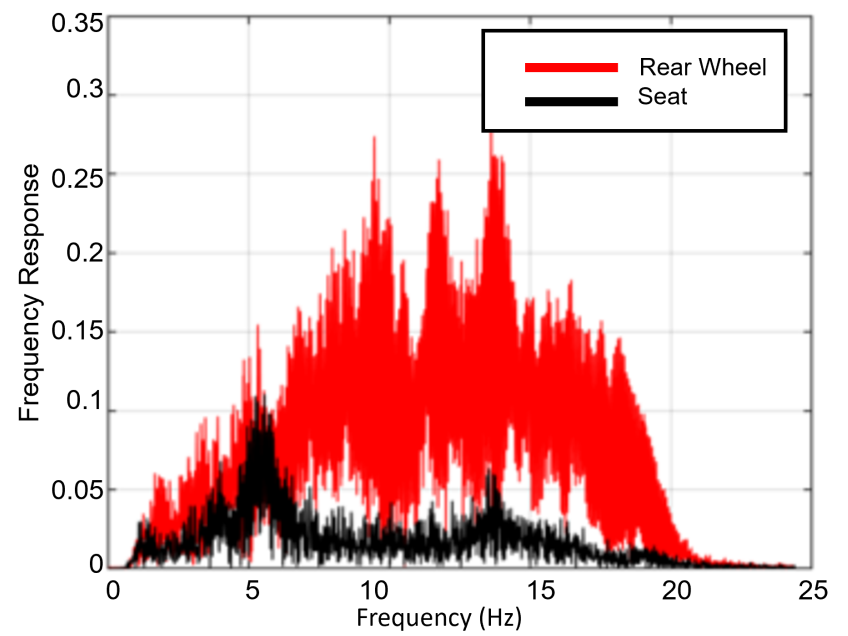

(c)

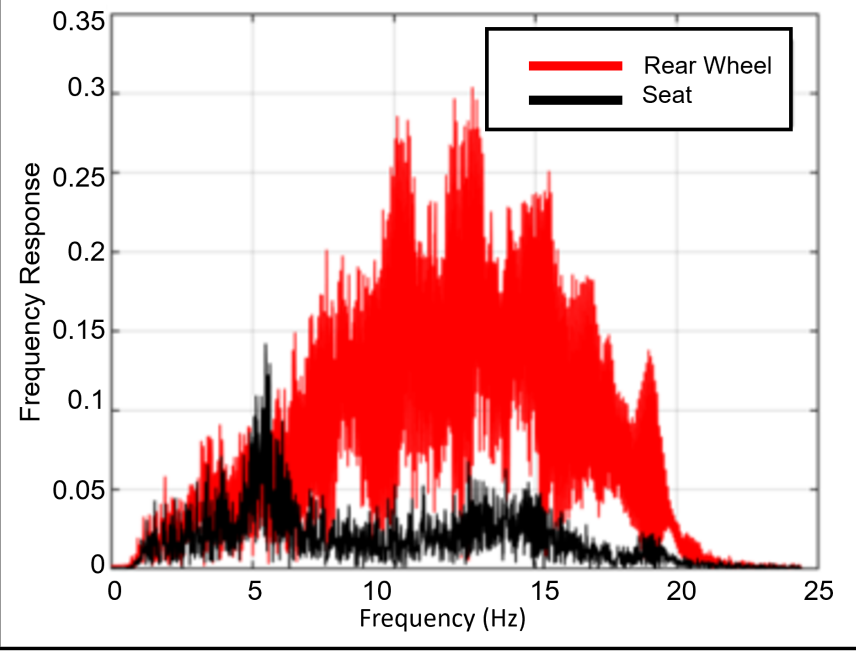

Figure 11. Frequency response - accelerations measured from sine sweep of $1-20 \mathrm{~Hz}$. (a) Current $=0.0 \mathrm{~A}$, (b) Current $=0.4 \mathrm{~A}$, (c) Current $=1.4 \mathrm{~A}$.

the seat post for the three different damper currents and also for the two control algorithms - on/off and proportional control. All the results in Fig. 12 were collected while testing the bike on a mountain trail, as explained in Section 2. While there is some variation in the data with the changing current, there is no clear effect of changing the damping current on the acceleration at the seat post. However, both control algorithms 


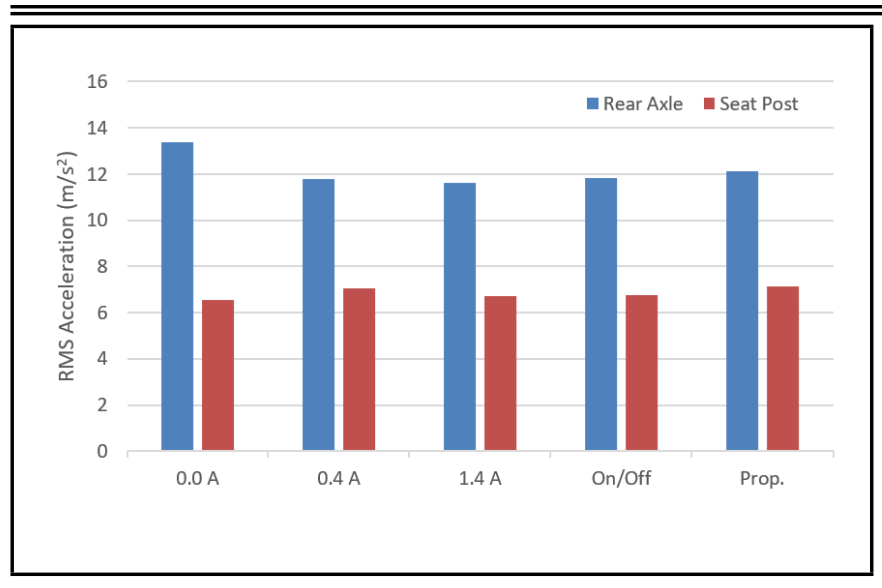

Figure 12. RMS accelerations - measured on the trail at three different damper currents, with the on/off control algorithm, and with the proportional control algorithm.
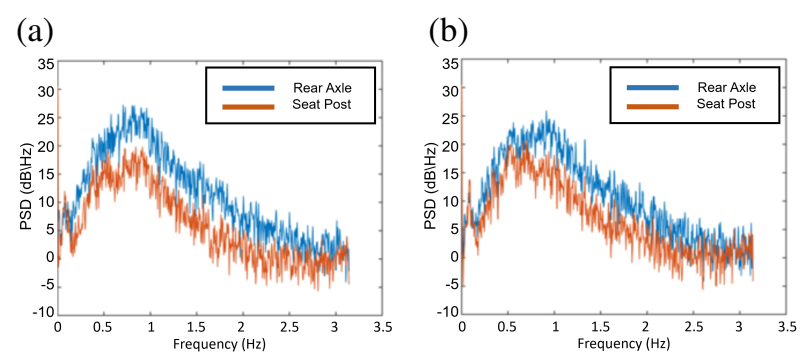

(c)

(d)
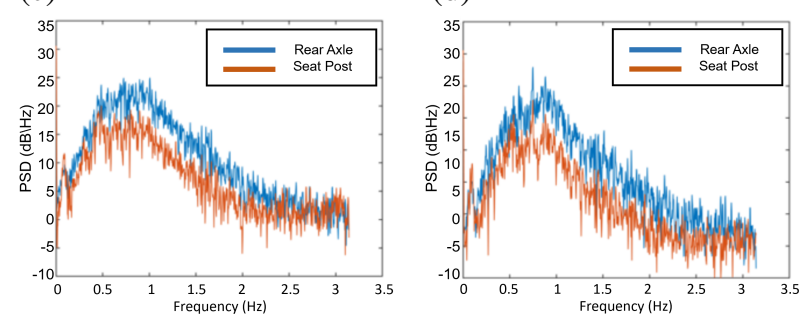

(e)

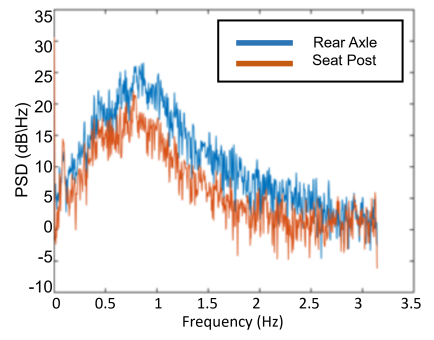

Figure 13. Power spectral density plots of acceleration data collected during trail testing. (a) Current $=0.0 \mathrm{~A}$, (b) Current $=0.4 \mathrm{~A}$, (c) Current $=1.4 \mathrm{~A}$ (d) On/off control, (e) Proportional control.

demonstrate a significant reduction in the acceleration at the seat post, as seen from Fig. 12.

The results from trail testing can be corroborated with the results shown in Fig. 13. Figure 13 shows the power spectral density (PSD) plots for acceleration at the seat post for the data collected on the trail. The capability of PSD to evaluate the influence of a random road profile is documented in the literature. ${ }^{19}$

Results from the acceleration PSD plots do not indicate any appreciable difference as the damper current is increased from
0 to $1.4 \mathrm{~A}$. The PSD plots at 0 A show slightly more separation between the rear axle and seat post plots than the $0.4 \mathrm{~A}$ and 1.4 A plots. This could be due to the stiffening of the suspension as the damper current is increased, however the difference seems to be relatively small. However, it may be noted that the amplitude of the PSD is reduced between the rear axle and seat post plots in all cases. The two control algorithms result in a similar reduction in amplitude, but the bandwidth is seen to slightly vary between the two results. This needs to be investigated further over different road profiles. Although the difference between the responses at different damper currents is clearly discerned from the shaker table test, such a clear distinction cannot be made for the results of trail testing. This could be due to the range of frequencies that gets excited on the mountain trail as compared to a specific frequency on the shaker table testing, and needs to be investigated further.

Some of the results from the theoretical model can be directly compared with the test results. For instance, the local peak of the sprung mass bounce acceleration PSD at $1 \mathrm{~Hz}$ is distinctly captured by the model. Furthermore, the transmissibility results of the sprung mass bounce and pitch can be directly compared to the frequency response from the sine sweep test. This indicates that the model can be developed further to simulate the control characteristics as well as variability associated with riding positions.

\section{CONCLUSIONS}

This study has provided the detailed design of a shock absorber for a mountain bike that utilizes an MR damper. Using commercially available air springs and an MR damper, a rear shock absorber has been developed and installed in a crosscountry style mountain bike. The damper has been experimentally evaluated on a shaker table as well as a mountain trail. Two control algorithms have been implemented to provide active damping. The damping level of the shock absorber can be controlled through a feedback control system that is implemented on a microcontroller and utilizes acceleration input from a MEMS accelerometer mounted at the rear axle of the bike. Since the control algorithm is implemented in the microcontroller program, it can be easily modified to accommodate a different logic based on rider preferences, for ride comfort or bike handling. The rear suspension system has been designed such that it can be used as a baseline to develop different control algorithms that can be used to tailor the response of the bike to different trail conditions and ride requirements. Experimental evaluation of the MR damper-based shock absorber on the shaker table indicates that the response of the bike can be directly controlled at relatively low frequency excitations through the input current to the electromagnet of the damper. This is useful in a mountain bike since the excitation frequency over a bumpy trail is typically below $5 \mathrm{~Hz}$. Shaker table testing above $5 \mathrm{~Hz}$ indicates that changing the input current does not significantly affect the seat post isolation even though the system still exhibits ride comfort by mitigating acceleration transmitted from the rear wheel.

The results of the preliminary mathematical model indicate that the power spectral density and transmissibility of the sprung mass bounce and pitch can be reasonably simulated to gain an insight into the control characteristics of the active 
damper. This model will need to be developed further to understand parameter sensitivity as well as rider variability.

In order to test the bike on a mountain trail, the system has been integrated to provide a portable design. Two control algorithms have been developed to implement an on-off control or a proportional control by using the acceleration data and by identifying the appropriate input current that should be used for the damper. Both control algorithms demonstrate significant levels of reduction of acceleration at the seat post, however it is necessary to test different control strategies in order to identify the right configuration for ride comfort and handling.

The main focus of this study has been on the design, integration, preliminary modeling, and testing of an active rear suspension system. Future work will focus on the development of several control strategies that can be used to provide ride comfort and handling performance, depending on the trail conditions, and compare the results to other studies in the literature. Evaluation of control strategies will be done by quantifying variables such as pedaling effort. The rear suspension system with active control will also be evaluated on multiple trails with different riders to get qualitative feedback and comprehend the influence of variables such as rider mass and inertia as well as rider positions. The preliminary model presented in this study will be developed further to integrate active control of the rear suspension system. This will allow a simulation of the response of the rear suspension system to different excitation inputs under varying riding conditions.

\section{REFERENCES}

1 Farrel, P. The Roots of Dirt: How Mountain Bikes Went from Clunkers to Global Phenomenon, Wired, www.wired.com/2016/06/ history-mountain-bike-unsurprisinglybadass/

2 Nielens, H., and Lejeune T. Energy Cost of Riding Bicycles with Shock Absorption Systems on a Flat Surface, International Journal of Sports Medicine, 22, 400-404, (2001). https://dx.doi.org/10.1055/s-2001-16242

3 Faiss, R., Praz, M., Meichtry, A., Gobelet, C., and Deriaz, O. The Effect of Mountain Bike Suspensions on Vibrations and Off-Road Uphill Performance, Journal of Sports Medicine and Physical Fitness, 47, 151-158, (2007).

4 MacRae, H., Hise, K., and Allen, P. Effects of Front and Dual Suspension Mountain Bike Systems on Uphill Cycling Performance, Medicine and Science in Sports \& Exercise, 32, 1276-1280, (2000). https://dx.doi.org/10.1097/00005768-200007000-00014

5 Nielens, H., and Lejeune, T. Bicycle Shock Absorption Systems and Energy Expended by the Cyclist, Sports Medicine, 34, 71-80, (2004). https://dx.doi.org/10.2165/00007256200434020-00001

6 McAndrews, M. Bicycle Shock Absorber with Slidable Inertia Mass, United States Patent 8,960,389.

7 Rome, D. Fox Live Valve Suspension: The Future is Now, Cycling tips, https://cyclingtips.com/2018/08/ fox-live-valve-electronically-controlled-suspension/
8 Batterbee, D. C., and Sims, N. D. Magnetorheological platform dampers for mountain bikes, Proc. SPIE 7290, Industrial and Commercial Applications of Smart Structures Technologies, 7290, 70-80, (2009). https://dx.doi.org/10.1117/12.811984

9 Pascoal, A., and Goncalves, J. Prototyping and Analysis of Comfort in Driving a Bicycle Equipped with a MagnetoRheological Damper, IEEE 6th Portuguese Meeting on Bioengineering, Lisbon, Portugal, Feb. 22-23, (2019). https://dx.doi.org/10.1109/enbeng.2019.8692561

10 Klug, S., Moia, A., Verhagen, A., Gorges, D., and Savearesi, S. Semi-Active Control on Bicycles: AntiDive during Road Excitation, IEEE Intelligent Vehicles Symposium, Paris, France, June 9-12, (2019). https://dx.doi.org/10.1109/ivs.2019.8813869

11 Breese, D. G., and Gordaninejad, F. Semi active field-controllable magneto-rheological fluid dampers for mountain bicycles, Proc. SPIE 3991, Smart Structures and Materials 2000: Industrial and Commercial Applications of Smart Structures Technologies, (2000). https://dx.doi.org/10.1117/12.388170

12 Carlson, J. D. MR Fluids and Devices in the Real World, International Journal of Modern Physics B, 19, 1463-1470, (2005). https://dx.doi.org/10.1142/S0217979205030451

13 Kaul, S. Recursive Modeling of a Magneto-Rheological Damper, International Journal of Mechanical and Materials Engineering, 6, 31-40, (2011).

14 Meeser, R. F., Kaul, S., and Els, P. S. Investigation into the Flow-Blocking Ability of a Novel Magneto-Rheological Damper Unit, ASTM Journal of Testing and Evaluation, 45, 1601-1608, (2017). https://dx.doi.org/10.1520/JTE20160053

15 Marzbanrad, J., Poozesh, P., and Damroodi, M. Improving vehicle ride comfort using an active and semi-active controller in a half-car model, Journal of Vibration and Control, 19, 1357-1377, (2012). https://dx.doi.org/10.1177/1077546312441814

16 Lord Technical Data: RD-8040-1 and RD 8041-1 Dampers, Lord Corporation, Cary, NC, USA, (2009).

17 Williams, T., Kaul, S., and Dhingra, A. Influence of Frame Stiffness and Rider Position on Bike Dynamics: Analytical Study, Proc. ASME International Mechanical Engineering Congress and Exposition, IMECE 2015, Houston, TX, (2015). https://dx.doi.org/10.1115/IMECE2015-50137

18 Kaul, S. Influence of a Vibration Isolation System on Planar Dynamics of a Motorcycle, International Journal of Acoustics and Vibration, 25, 96-103, (2020). https://dx.doi.org/10.20855/ijav.2020.25.11603

19 Sun, L. On Human Perception and Evaluation to Road Surfaces, Journal of Sound and Vibration, 247, 547-560, (2001). https://dx.doi.org/10.1006/jsvi.2001.3642 